\title{
Comparison of a hydrogel corneal inlay and monovision laser in situ keratomileusis in presbyopic patients: focus on visual performance and optical quality
}

\author{
This article was published in the following Dove Press journal: \\ Clinical Ophthalmology \\ 20 September 2017 \\ Number of times this article has been viewed
}

\section{Cornelis Verdoorn \\ Lasik Centrum Eye Clinic, Boxtel, the Netherlands}

Correspondence: Cornelis Verdoorn Lasik Centrum Eye Clinic, Bosscheweg 149, 5282wv, Boxtel, the Netherlands Tel +003 I 6I I73 II83 Email c.verdoorn@bergmanclinics.nl
Purpose: To compare the visual performance and optical quality after Raindrop Near Vision Inlay implantation or monovision LASIK for the correction of presbyopia.

Materials and methods: In this retrospective case-series study, patients previously treated in the nondominant eye with monovision LASIK were compared with patients previously implanted with Raindrop Near Vision Inlay. The study enrolled 16 inlay and 15 monovision LASIK patients. Uncorrected near visual acuity, uncorrected distance visual acuity, binocular stereopsis, patient satisfaction, and patient task performance were assessed.

Results: Postoperatively, the mean spherical equivalent was $-0.66 \mathrm{D}(0.78 \mathrm{SD})$ for the inlay group and $-1.03 \mathrm{D}(0.56 \mathrm{SD})$ for the monovision LASIK group. Monocularly, at uncorrected near distances, $60 \%$ of inlay patients and $47 \%$ of monovision LASIK patients achieved $\geq 20 / 20$. Monocularly, at uncorrected far distances, $75 \%$ of inlay patients and $40 \%$ of monovision LASIK patients achieved $\geq 20 / 32$ vision. Binocularly, at near distances, $79 \%$ of inlay patients and $53 \%$ of monovision LASIK patients obtained $\geq 20 / 20$ vision. All patients achieved $\geq 20 / 20$ binocularly for distance. On average, inlay patients obtained 98 seconds of arc and monovision LASIK patients obtained 286 seconds of arc for stereopsis. Most (79\%) of the inlay patients and 66\% of monovision LASIK patients were satisfied with their near vision, while $86 \%$ of inlay patients and $67 \%$ of monovision LASIK patients were satisfied with their distance vision.

Conclusion: Patients receiving corneal inlays demonstrated better near and distance visual acuities, binocular stereopsis, task performance, and satisfaction, when compared to patients treated with monovision LASIK.

Keywords: presbyopia, corneal inlays, monovision, laser in situ keratomileusis, LASIK, Raindrop Near Vision Inlay

\section{Introduction}

Presbyopia affects everyone over the age of 50, and as this particular patient population continues to grow, the need for surgical and nonsurgical treatment options will grow as well. ${ }^{1}$ The correction of presbyopia in emmetropic patients represents a challenge for refractive surgeons today. Due to this need, different presbyopia correcting products and treatments have been introduced to eliminate or reduce the dependence on reading glasses. Current surgical and nonsurgical treatments for presbyopia include monovision with contact lens or LASIK, presbyopia-correcting intraocular lenses, presbyLASIK, and corneal inlays. The most common technique used to treat presbyopia is a monovision approach. ${ }^{3}$ Monovision induces slight anisometropia with the nondominant eye targeted 
for near vision and the dominant eye set for distance vision. ${ }^{4}$ This technique does not work well for everyone and often requires patients with good blur suppression to ensure a high rate of success. ${ }^{5}$ In addition, compromises in binocular visual acuity, stereoacuity, contrast sensitivity, task performance, and depth of focus must be taken into consideration. ${ }^{5}$

Another surgical approach to presbyopia treatment is the use of corneal inlays. ${ }^{5}$ These devices, initially developed in the 1940 s by Barraquer, ${ }^{6}$ are relatively small, with a diameter of 2-4 mm. ${ }^{6}$ Corneal inlays are implanted on the corneal stroma under a corneal flap or within a corneal pocket and correct presbyopia through various mechanisms of action. ${ }^{7}$ The Kamra Inlay (Acufocus, Irvine, CA, USA) uses the pinhole principle to increase the depth of focus, the Flexivue Microlens (Presbia, Irvine, CA, USA) uses a refractive annular add to create a zone for near vision, and the Raindrop Near Vision Inlay (ReVision Optics, Inc., Lake Forest, CA, USA) changes the curvature of the anterior cornea, which increases the refractive power at the center of the pupil. ${ }^{8}$

The Raindrop Inlay is made of a clear permeable hydrogel material that has a diameter of $2.0 \mathrm{~mm}$ and a central thickness of $\sim 30 \mu \mathrm{m} .^{9}$ The refractive index of the device is similar to that of the surrounding corneal tissue (1.376). ${ }^{6}$ The shape of the inlay is thicker at the center and thinner at the edges, creating a near-power effect in the center of the pupil and gradually transitioning to an unaltered cornea in the midperiphery. ${ }^{10}$ In addition, the inlay is implanted without removing corneal tissue. Therefore, its refractive effect can be reversed by removal of the inlay. ${ }^{1,11}$

Following implantation of the Raindrop Inlay, the final shape of the anterior cornea is affected by remodeling of both the overlying anterior flap and the epithelium. ${ }^{6}$ A prospective study in 30 emmetropic presbyopic patients demonstrated the epithelium remodels within a zone about twice the diameter of the inlay. ${ }^{12}$ In that study, the central anterior corneal surface rose by an average of $9.8 \mu \mathrm{m}$, providing a progressive add power profile. This change in anterior corneal height provides for better near and intermediate vision. The relationship between the epithelial, stromal, and anterior corneal surface changes that were observed in this study indicate that epithelial changes are greatest in the corneal regions with greater local surface curvature.

Recent studies have demonstrated that patients receiving the Raindrop Inlay experienced improvements in both near and intermediate visual acuity. ${ }^{7,13-15}$ Garza et al, ${ }^{7}$ Chayet and Barragan Garza, ${ }^{14}$ and Whitman et $\mathrm{al}^{15}$ reported that Raindrop is safe and effective in emmetropes, myopes, and hyperopes. One year results from a large multicenter study in emmetropic patients showed that $93 \%$ of patients achieved uncorrected near visual acuity (UNVA) of $\geq 20 / 25$, with similar results obtained for uncorrected intermediate visual acuity and uncorrected distance visual acuity (UDVA). ${ }^{15}$ Moreover, binocular mean UDVA exceeded 20/20 (3 months through 1 year). Most patients (96\%) reported absent or mild scores for visual symptoms (glare, halos, double vision, and fluctuations in vision), ocular symptoms (99\%; pain, light sensitivity, and discomfort), and dryness (95\%). Adverse events resolved with treatment. Recent results with the inlay demonstrated refractive stability was achieved within 6 months and was maintained for 24 months. ${ }^{16}$

A dioptric power range of -0.5 to $+1.5 \mathrm{D}$ before implantation appears to be ideal to create balanced visual outcomes. ${ }^{10}$ A study in emmetropic patients with +1.50 to $+2.00 \mathrm{D}$ of presbyopia reported $97 \%$ of patients who received the inlay achieved visual acuities of $0.2 \log$ MAR (20/32) or better at near, intermediate, and far distances. ${ }^{13}$ One year after implantation, distance-corrected near acuity improved by more than 3 lines. Patients achieved a distance-corrected visual acuity of $0.3 \log$ MAR (20/40) or better across a $3.50 \mathrm{D}$ range of defocus. The average reduction in the reading add was $1.60 \mathrm{D}$ overall. There were no significant changes in the patients' binocular contrast sensitivity following implantation. In addition, this study and others have shown presbyopic patients generally have a high level of satisfaction with the hydrogel inlay. ${ }^{13,14,17}$

In this study, we compared the visual performance, stereopsis, task performance, and satisfaction of patients previously treated with the Raindrop Near Vision Inlay with those who received monovision LASIK for the correction of presbyopia.

\section{Materials and methods}

Verbal informed consent was obtained from each patient before any procedures were performed. Since this study was retrospective in nature, it did not fall under the Medical Research Involving Human Subjects Act. Therefore, the study did not require approval by a Medical Research and Ethics Committee or the Centrale Commissie Mensgebonden Onderzoek.

\section{Study design}

The study was conducted in a private LASIK clinic in Boxtel, the Netherlands. This was a nonrandomized, retrospective case-series study comparing patients previously treated with two different types of surgical correction for presbyopia. All data were collected at a single time point. The patients were 
evaluated at a time point in which their vision would be considered stable, 4 months after surgery or later.

\section{Surgical technique}

Raindrop Inlays were previously implanted in the nondominant eyes. A flap with a diameter of $8.0 \mathrm{~mm}$ and a depth of $150 \mu \mathrm{m}\left(30 \%\right.$ central corneal thickness recommended $\left.{ }^{18}\right)$ was created using femtosecond laser IntraLase $150 \mathrm{iFS} 150 \mathrm{kHz}$ (Johnson and Johnson Vision Care, Santa Ana, CA, USA). Patients with residual refractive error were treated with WaveLight Allegretto Eye-Q $400 \mathrm{~Hz}$ (Alcon Laboratories, Inc., Fort Worth, TX, USA) to target refraction of plano for the dominant eye and $+0.75 \mathrm{D}$ to the nondominant eye prior to inlay implantation (this is off label in the USA). After lifting the flap, the inlay was then delivered on the stromal bed using a preloaded inserter according to the manufacturer's instructions. The inlay was positioned over the center of the light-constricted pupil and allowed to dry for $\sim 1$ minute before the flap was replaced on the corneal bed.

Monovision LASIK procedures were performed under topical anesthesia using IntraLase to create the corneal flap and the WaveLight Excimer laser for ablation. All nondominant eyes were corrected for near vision (targeted myopia -0.75 to $-1.25 \mathrm{D})$. The dominant eye was targeted for plano.

\section{Testing}

All patients had both eyes best corrected to 20/20 preoperatively. Monocular and binocular uncorrected visual acuities were measured for near at $40 \mathrm{~cm}$ (UNVA) and for distance at $6 \mathrm{~m}$ (UDVA) using a Snellen visual acuity chart. Stereo acuities were measured uncorrected at $40 \mathrm{~cm}$ using the Randot Stereo Test (Stereo Optical Co., Inc., Chicago, IL, USA). A subjective questionnaire was used to collect visual tasks performance and patient satisfaction. Subjective questions were targeted for near and distance vision, with options being very satisfied, satisfied, neutral, dissatisfied, and very dissatisfied. Patients were also asked about task performance at different distances without using a correction in good and dim light. The answers were scored as follows: $0=$ not at all; $1=$ with difficulty; $2=$ with ease. $^{7}$ The points were added to get a cumulative task score with a maximum score of 10 . Table 1 shows the tasks at different distances. All results were compared in both groups.

\section{Statistical methods}

The statistical analyses were performed using Microsoft Excel software. Data were reported as means and analyzed
Table I Patient subjective task assessment

\begin{tabular}{|c|c|c|}
\hline \multicolumn{3}{|c|}{ Asked the patient if they can } \\
\hline Near tasks & Intermediate tasks & Distance tasks \\
\hline $\begin{array}{l}\text { Read medicine } \\
\text { instructions }\end{array}$ & $\begin{array}{l}\text { Find items on a kitchen } \\
\text { shelf }\end{array}$ & Read street signs \\
\hline Read newspaper & Read a computer screen & $\begin{array}{l}\text { Identify people } \\
\text { across a room }\end{array}$ \\
\hline $\begin{array}{l}\text { Examine their } \\
\text { fingernails }\end{array}$ & Use a bathroom mirror & Judge car distances \\
\hline Dial a cell phone & Use a wall calendar & Read house numbers \\
\hline Read a magazine & $\begin{array}{l}\text { Recognize framed photo } \\
\text { portraits }\end{array}$ & $\begin{array}{l}\text { Tell the time from a } \\
\text { wall clock }\end{array}$ \\
\hline
\end{tabular}

using a Student's $t$-test ( $P$-values $<0.05$ for statistical significance). Note this alpha of 0.05 was not modified for multiple testing by the Bonferroni correction. Error bars denote standard errors (SE).

\section{Results}

The average follow-up period for the inlay implantation was 9 months and for monovision LASIK was 32 months.

The age range of the inlay group was between 45 and 62 years (mean age 53 years). There were 11 females and 5 males in this treatment group. Emmetropic and low hyperopic patients $(n=6)$ received only the inlay. They had an average preoperative spherical equivalent refraction of $+0.33 \mathrm{D}(+0.00$ to $+0.50 \mathrm{D})$. Hyperopic patients $(n=10)$ with an average preoperative spherical equivalent refraction of $+1.54 \mathrm{D}(-0.125$ to $+3.750 \mathrm{D})$ received a treatment of LASIK combined with an inlay. The LASIK treatment in the nondominant eye was targeted for +0.75 with zero cylinder. The dominant eye was treated for plano at distance.

The second group comprised monovision LASIK patients $(n=15)$ with age ranging between 49 and 66 years (mean age 56 years). There were nine females and six males in this study group. The average preoperative spherical equivalent refraction of this group was $+1.28 \mathrm{D}(+0.00$ to $+2.50 \mathrm{D})$. LASIK was performed in both eyes in most patients $(n=11)$. The dominant eye was corrected for distance.

Posttreatment mean spherical equivalent was $-0.66 \mathrm{D}$ $(\mathrm{SD}=0.78)$ in the inlay eye and $+0.05 \mathrm{D}(\mathrm{SD}=0.34)$ in the fellow eye, while monovision LASIK was $-1.03 \mathrm{D}(\mathrm{SD}=0.56)$ for the nondominant eye and $+0.26 \mathrm{D}(\mathrm{SD}=0.48)$ for the dominant eye.

\section{Clinical outcomes} Monocular assessment

Corneal inlay patients achieved better UNVA in the nondominant eye: $64 \%$ of the inlay and $47 \%$ of the monovision 

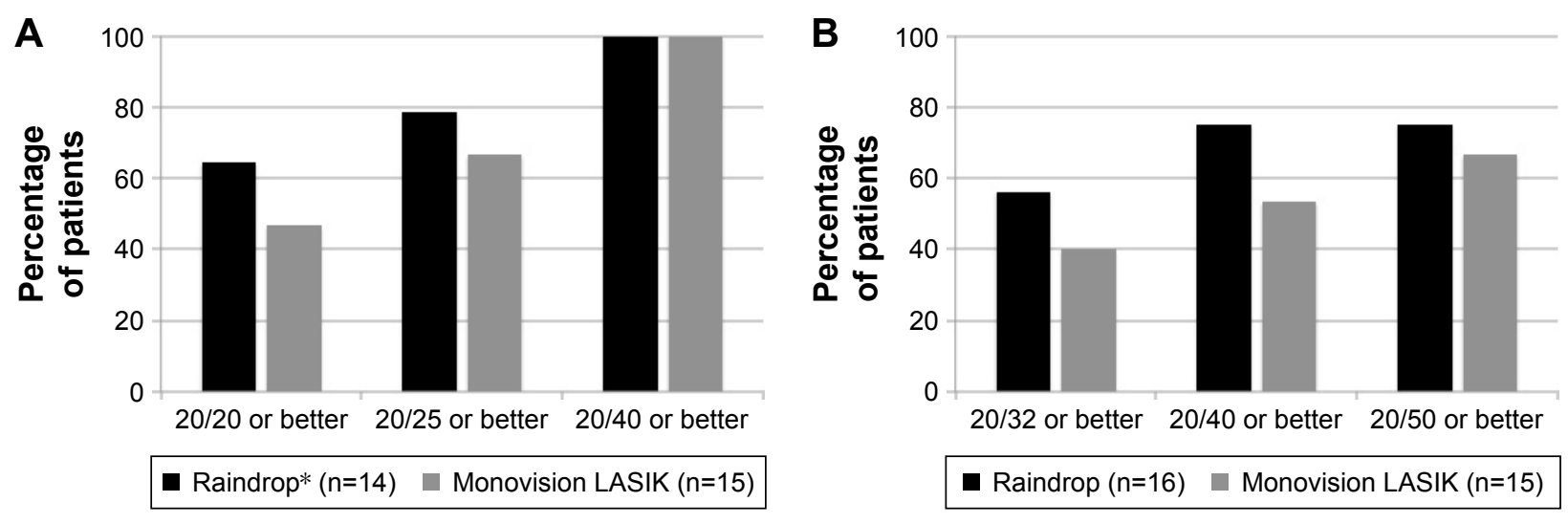

Figure I (A) Near uncorrected and (B) distance uncorrected monocular visual acuity for Raindrop and monovision LASIK patients. Note: *Two patient data points were missing in the Raindrop near group.

LASIK patients achieved 20/20 vision or better. All patients of both groups achieved 20/40 vision or better. In terms of distance vision, $56 \%$ of the inlay patients and $40 \%$ of the monovision LASIK patients achieved 20/32 vision or better in the nondominant eye (Figure 1A and B).

\section{Binocular assessment}

All patients achieved 20/32 or better for near vision, with $81 \%$ of the inlay patients and $53 \%$ of the monovision LASIK patients achieving 20/20 or better. For distance, $100 \%$ of inlay patients and $87 \%$ of monovision LASIK patients achieved 20/20 vision or better (Figure 2A and B).

Stereoacuity results, on average, were statistically better in the Raindrop group when compared to the monovision LASIK group (Raindrop Inlay: 98 seconds of arc [SE =26] versus monovision LASIK: 286 seconds of arc [SE $=66.7$; $P<0.05]$ ), as shown in Figure 3.

\section{Task assessment}

Task assessment questions were asked as shown in Table 1. In good light conditions, both groups were able to perform tasks with ease at both distances. For distance, inlay patients achieved 9.8 ( $\mathrm{SE}=0.12$ ) compared with monovision LASIK patients who achieved 9.6 ( $\mathrm{SE}=0.16)$. For near, inlay patients achieved a score of $9.1(\mathrm{SE}=0.27)$ and monovision LASIK patients achieved a score of 8.5 ( $\mathrm{SE}=0.38$; Figure 4A). There was no statistically significant difference between the two groups.

In dim light conditions, both groups easily performed distance tasks, but had more difficulty with near tasks. At distance, inlay patients achieved a score of $9.7(\mathrm{SE}=0.14)$ and monovision LASIK patients achieved a similar score of 9.5 ( $\mathrm{SE}=0.18)$. For near, inlay patients and monovision LASIK patients obtained scores of $8.3(\mathrm{SE}=0.34)$ and 7.6 $(\mathrm{SE}=0.38)$, respectively (Figure 4B). There was no statistically significance difference between the two groups.

\section{Patient satisfaction}

Most (79\%) of the inlay patients and $67 \%$ of the monovision LASIK patients reported that they were satisfied or very satisfied with their near vision (Figure 5A). For distance vision, $86 \%$ of the inlay patients and $67 \%$ of the monovision LASIK
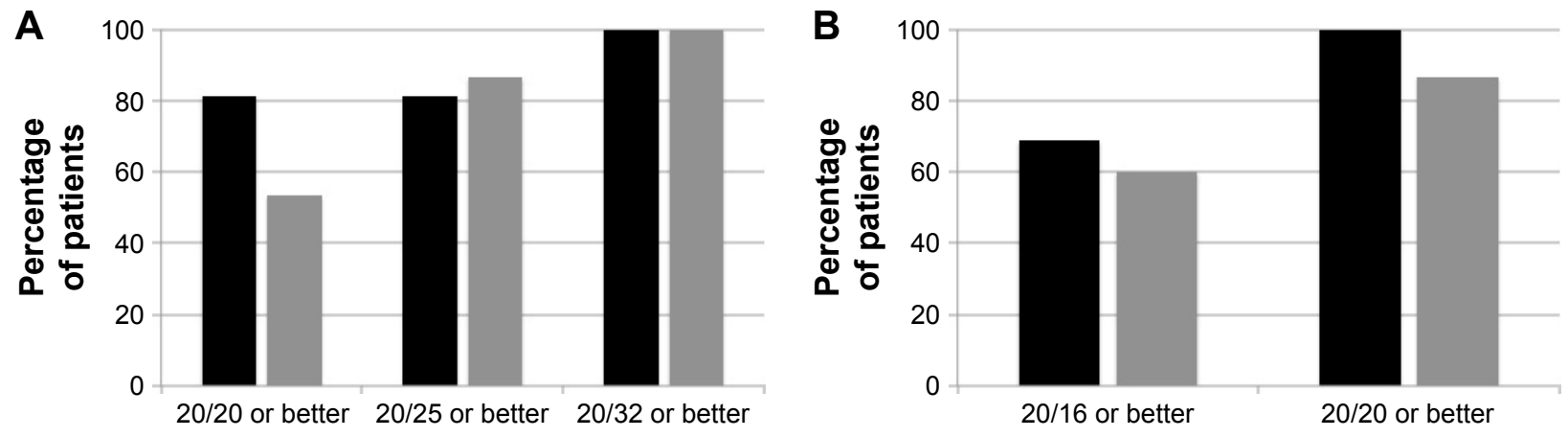

Raindrop $(n=16) \quad$ Monovision LASIK $(n=15)$

Figure 2 (A) Near uncorrected and (B) distance uncorrected binocular visual acuity for Raindrop and monovision LASIK patients. 


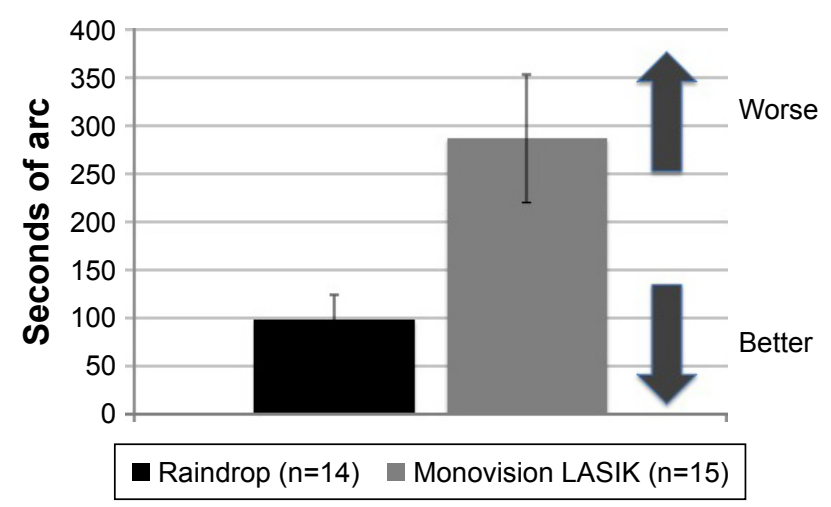

Figure 3 Stereoacuity in seconds of arc for Raindrop and monovision LASIK groups

patients reported being satisfied or very satisfied. None of the inlay patients were dissatisfied with their distance vision (Figure 5B). Six monovision LASIK patients were retreated due to visual complaints (two for near and four for distance). All retreated patients were counted as dissatisfied.

\section{Discussion}

Patients receiving the Raindrop Inlay achieved better uncorrected visual acuity both at near and far distances in the nondominant eye than those treated with monovision LASIK. Higher proportions of patients obtained 20/20 vision or better with the corneal inlay, compared to monovision LASIK treatment. The corneal inlay patients also obtained better results in terms of binocular stereoacuity, compared with those receiving monovision LASIK. Patients from the two treatment groups showed comparable abilities to perform tasks at near and far distances in good lighting conditions. Under dim lighting conditions, patients from both groups performed distance tasks well, but had some difficulty with near tasks. Finally, higher proportions of inlay patients were satisfied or very satisfied with their near and distance vision, compared with the monovision LASIK patients. Taken together, these results suggest that visual acuity and patient satisfaction with the corneal inlay were comparable or better than those with monovision LASIK.

Monovision LASIK is known to cause side effects that are a direct result of imbalance or anisometropia. ${ }^{19}$ These adverse effects include: blur in near or distance vision, halos and glare (particularly at night), diminished vision at night, reduced depth perception, or even transient diplopia caused by temporary strabismus. Alarcon et al evaluated outcomes in presbyopic monovision LASIK patients and found that all patients had significantly worse stereoacuity $(P<0.001){ }^{20}$ Nine (39\%) of these patients achieved stereoacuities between 40 and 200 arc of second, while the remaining patients had stereoacuities of $\geq 800 \mathrm{arc}$ of second. Another study reported small decreases in both stereopsis and contrast sensitivity in patients with monovision LASIK correction versus full distance correction. ${ }^{21}$ Reilly et al found the distance eye had to be corrected within very small tolerance limits in monovision LASIK patients. ${ }^{22}$ Their reported enhancement rate was $21 \%$ for the distance eye, but only $7 \%$ for the near eye.

Success with monovision treatment often depends on the amount of addition given to the nondominant eye. Durrie ${ }^{23}$ reported that near vision in the "near" eye improves with increased power, but with a stronger compromise to distance vision in the same eye. While monovision LASIK patients in this study were targeted for myopia (from -0.75 to $-1.25 \mathrm{D}$ ) in the nondominant eye in order to increase the depth of field, inlay patients were targeted for $+0.75 \mathrm{D}$, as a targeted range of -0.5 to $+1.5 \mathrm{D}$, for inlay patients, has been clinically shown to achieve better balanced vision due to extended depth of focus for light rays at the center of the pupil. ${ }^{10}$ While the
A

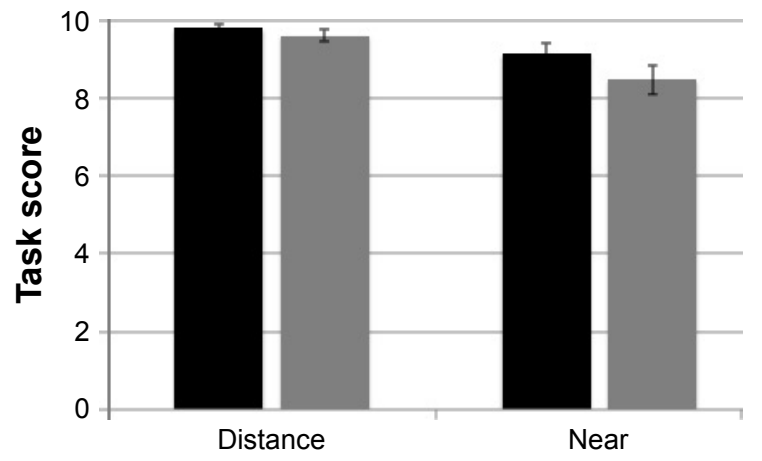

B

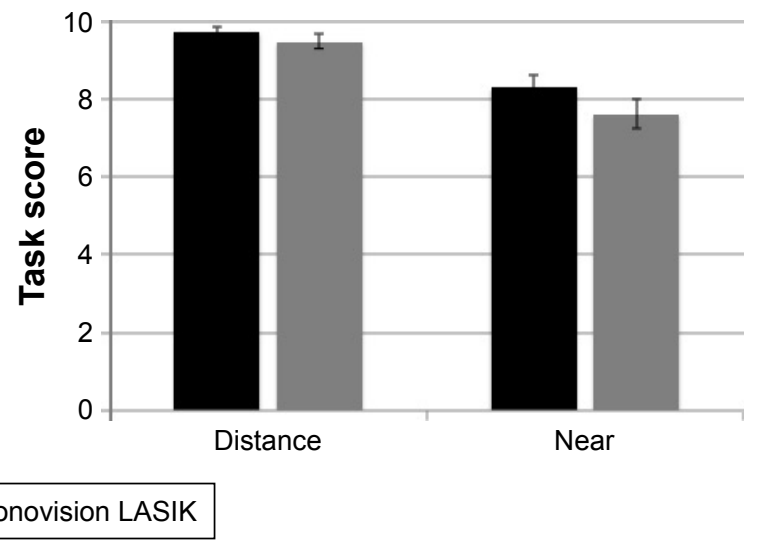

Figure 4 (A) Patient assessment of ease of performing visual tasks in good light. (B) Patient assessment of ease of performing visual tasks in dim light. 


\section{A Monovision \\ LASIK ( $n=15)$}

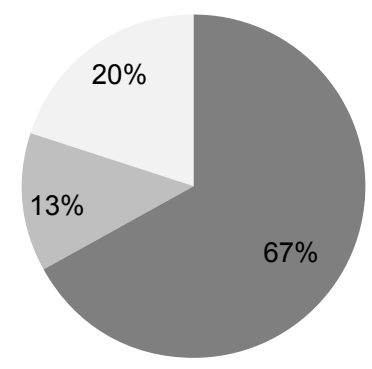

\section{B Monovision LASIK $(n=15)$}

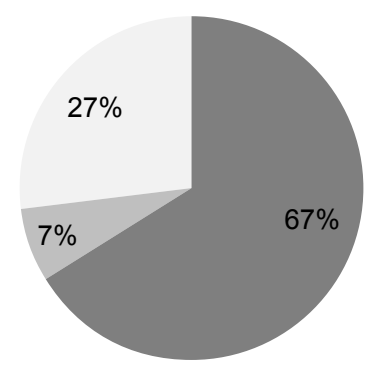

Satisfied or very satisfied Neutral Dissatisfied or very dissatisfied

Figure 5 Raindrop and monovision LASIK satisfaction for (A) near and (B) distance vision.

Note: Retreated monovision LASIK patients were counted as dissatisfied.

postop refraction was similar for Raindrop $(-0.66 \mathrm{D})$ patients and for monovision LASIK ( $-1.03 \mathrm{D})$ patients in the nondominant eye, the inlay group experienced better visual acuity as well as stereopsis. This was due to the Raindrop Inlay creating a progressive refractive power on the cornea that provides continuous near to distance vision.

Another study assessed the independence of visual performance in presbyopic patients who received the Raindrop Inlay in the nondominant eye. ${ }^{10}$ UDVAs following implantation were weakly associated with preoperative manifest refraction spherical equivalents. The mean preoperative uncorrected near, intermediate, and distance visual acuities were $20 / 25,20 / 25$, and $20 / 32$, respectively. The study demonstrated good visual acuity and patient task performance with the inlay from near through distance over a $2.0 \mathrm{D}$ range of preoperative refraction and patients' age ranging from 45 to 60 years.

Raindrop patients also showed similar trends with higher visual satisfaction at distance and near, compared to the monovision LASIK in this study. Few (7\%) of the inlay patients were unsatisfied with the near vision and none were dissatisfied with their distance vision. Approximately $20 \%$ of the monovision LASIK group was unsatisfied with their distance vision and $27 \%$ was unsatisfied with their near vision.

Several studies have shown that monovision LASIK patients tend to exhibit higher retreatment rates, especially for distance vision. ${ }^{2}$ Monovision patients typically use their dominant eye for distance vision, and thus, very precise outcomes are required. ${ }^{5,24}$ In this study, six monovision LASIK patients were retreated because of dissatisfaction: $27 \%$ needed retreatment for distance vision and $13 \%$ needed retreatment for near vision. Age is a factor that significantly increases the risk for retreatment following laser refractive surgery. ${ }^{25}$ In older patients, a myopic end point is desirable due to the decrease in accommodative amplitude; ${ }^{26}$ therefore, monovision LASIK patients may require retreatment more often, whereas the Raindrop Inlay changes the shape of the cornea to create a smooth gradient of power without sharp transitions. In addition, the Inlay is an additive technology, which can be removed if necessary. In contrast, LASIK requires the removal of corneal tissue, which cannot be reversed.

Garza and Chayet $^{17}$ have pointed out that the refractive effect of the Raindrop Inlay in the nondominant eye is different from the effect of monovision LASIK without inlay implantation in the dominant eye. Unlike monovision, the corneal implant creates a multifocal cornea that is able provide near through distance vision. ${ }^{7}$ In an eye treated with the inlay, the peripheral region of the cornea is still relatively emmetropic. Distance vision in an eye receiving the implant does not decrease to the extent of eyes treated with monovision. Therefore, the nondominant eye retains good distance vision. Even monocularly, the distance visual acuity is good for patients receiving the Raindrop Inlay due to the continuous center-near power profile of the device. ${ }^{10}$ In contrast, the monovision approach tends to cause enough defocus in the treated eye to diminish binocular vision. ${ }^{23}$

The largest difference between the two treatment groups was observed in binocular function. The reduction of stereopsis in monovision is well documented in published studies. Stronger reading addition often leads to a corresponding loss in stereoacuity. ${ }^{5,27}$ Correcting one eye for near and one eye for far vision results in loss of stereoacuity, which some patients may have trouble with, especially if their occupation requires good binocularity. ${ }^{20}$ The mean difference in postop manifest refraction spherical equivalent between the nondominant and dominant eye was $0.72 \mathrm{D}$ for the Raindrop group and $1.29 \mathrm{D}$ for the monovision LASIK group. Because monovision has a higher 
amount of disparity between the two eyes, this results in a consequent reduction in binocular visual acuity and stereopsis. ${ }^{28}$

Corneal inlays have produced stable visual outcomes for at least 3 years following implantation. ${ }^{29}$ One prospective study in myopic patients with presbyopia reported that 1 year after concurrent bilateral LASIK with inlay implantation in the nondominant eye, $98 \%$ of all visual tasks were easily performed without vision correction. ${ }^{17}$ Furthermore, 90\% of the patients were either satisfied or very satisfied with their vision overall. Another study found 100\% (19/19) of eyes achieved UNVA of 0.2 logMAR (20/32 Snellen) or better in the operative eye at 1 year.

Yoo et $\mathrm{al}^{30}$ reported improvements in UCVA in Korean patients with presbyopia who received the Raindrop Inlay. They found the preoperative monocular UNVA of the eyes receiving the inlay was 20/129 \pm 1 Snellen and improved to $20 / 35 \pm 2$ Snellen $(P<0.01)$ at 6 months after implantation. Approximately $82 \%$ of the patients were satisfied or very satisfied with their vision overall. These results are comparable to those from this study in terms of visual acuity and patient satisfaction, where $79 \%$ and $86 \%$ of the patients were satisfied or very satisfied with their near vision and distance vision, respectively.

The difference in follow-up time between the two treatment groups is a limitation of this study. The average follow-up was 9 months postoperative for the inlay and 32 months postoperative for monovision LASIK. Differences in follow-up timing could give different outcomes as adaptation improves with time; however, studies show that Raindrop patients achieve refractive stability by 6 months ${ }^{15}$ and micro-monovision LASIK patients achieve stability from 6 weeks. $^{31}$

This study was limited by its short follow-up time. In addition, safety data were not collected. However, previous studies have shown a favorable safety profile for patients receiving the inlay. ${ }^{13,15}$

Our study showed that both Raindrop Inlay and monovision LASIK treatments are effective surgical options to treat presbyopia. With both treatments, patient selection and information are critical to successful outcomes. An accurate preoperative examination is especially needed in monovision LASIK to prevent postoperative disruptions in binocular vision. Raindrop patients demonstrated comparable or better improved visual acuities, binocular function, task performance, and visual satisfaction, when compared to patients in the monovision LASIK group. Additionally, the Raindrop Inlay can be removed if needed. Future prospective studies are needed to corroborate these results.

\section{Acknowledgment}

This study was supported by ReVision Optics, Inc., Lake Forest, CA, USA.

\section{Disclosure}

Dr Verdoorn has served as a consultant for ReVision Optics, Inc. The author reports no other conflicts of interest in this work.

\section{References}

1. Lindstrom RL, Macrae SM, Pepose JS, Hoopes PC Sr. Corneal inlays for presbyopia correction. Curr Opin Ophthalmol. 2013;24(4):281-287.

2. Reinstein DZ, Carp GI, Archer TJ, Gobbe M. LASIK for presbyopia correction in emmetropic patients using aspheric ablation profiles and a micro-monovision protocol with the Carl Zeiss Meditec MEL 80 and VisuMax. J Refract Surg. 2012;28(8):531-541.

3. Arba Mosquera S, Alio JL. Presbyopic correction on the cornea. Eye Vis (Lond). 2014;1:5.

4. Zheleznyak L, Sabesan R, Oh JS, MacRae S. Modified monovision with spherical aberration to improve presbyopic through-focus visual performance. Invest Ophthalmol Vis Sci. 2013;54(5):3157-3165.

5. Braun EH, Lee J, Steinert RF. Monovision in LASIK. Ophthalmology. 2008;115(7):1196-1202.

6. Whang WJ, Yoo YS, Joo CK, Yoon G. Changes in keratometric values and corneal high order aberrations after hydrogel inlay implantation. Am J Ophthalmol. 2017;173:98-105.

7. Garza EB, Gomez S, Chayet A, Dishler J. One-year safety and efficacy results of a hydrogel inlay to improve near vision in patients with emmetropic presbyopia. J Refract Surg. 2013;29(3):166-172.

8. Guell JL, Barrada OA. Intracorneal inlays - special focus on the raindrop. Eur Ophthalmic Rev. 2014;8(1):23-30.

9. Pinsky PM. Three-dimensional modeling of metabolic species transport in the cornea with a hydrogel intrastromal inlay. Invest Ophthalmol Vis Sci. 2014;55(5):3093-3106.

10. Steinert RF, Schwiegerling J, Lang A, et al. Range of refractive independence and mechanism of action of a corneal shape-changing hydrogel inlay: results and theory. J Cataract Refract Surg. 2015;41(8): 1568-1579.

11. Arlt E, Krall E, Moussa S, Grabner G, Dexl A. Implantable inlay devices for presbyopia: the evidence to date. Clin Ophthalmol. 2015;9: 129-137.

12. Lang AJ, Holliday K, Chayet A, Barraga-Garza E, Kathuria N. Structural changes induced by a corneal shape-changing inlay, deduced from optical coherence tomography and wavefront measurements. Invest Ophthalmol Vis Sci. 2016;57(9):OCT154-161.

13. Whitman J, Hovanesian J, Steinert RF, Koch D, Potvin R. Throughfocus performance with a corneal shape-changing inlay: One-year results. J Cataract Refract Surg. 2016;42(7):965-971.

14. Chayet A, Barragan Garza E. Combined hydrogel inlay and laser in situ keratomileusis to compensate for presbyopia in hyperopic patients: one-year safety and efficacy. J Cataract Refract Surg. 2013;39(11): 1713-1721.

15. Whitman J, Dougherty PJ, Parkhurst GD, et al. Treatment of presbyopia in emmetropes using a shape-changing corneal inlay: one-year clinical outcomes. Ophthalmology. 2016;123(3):466-475.

16. Frellick M. Corneal inlays better than monovision, researchers say. Medscape Medical News; October 26, 2016. Available at: http://www. medscape.com/viewarticle/871004. Accessed March 8, 2017.

17. Garza EB, Chayet A. Safety and efficacy of a hydrogel inlay with laser in situ keratomileusis to improve vision in myopic presbyopic patients: one-year results. J Cataract Refract Surg. 2015;41(2):306-312.

18. Raindrop Near Vision Inlay Professional Use Information. 2017. ReVision Optics, Inc. Lake Forest, CA. 
19. Pieramici S, Feldman BH. American Academy of Ophthalmology. Monovision LASIK. Available from: http://eyewiki.aao.org/ Monovision_LASIK. September 2014. Accessed March 8, 2017.

20. Alarcon A, Anera RG, Villa C, Jimenez del Barco L, Gutierrez R. Visual quality after monovision correction by laser in situ keratomileusis in presbyopic patients. J Cataract Refract Surg. 2011;37(9):1629-1635.

21. Garcia-Gonzalez M, Teus MA, Hernandez-Verdejo JL. Visual outcomes of LASIK-induced monovision in myopic patients with presbyopia. Am J Ophthalmol. 2010;150(3):381-386.

22. Reilly CD, Lee WB, Alvarenga L, Caspar J, Garcia-Ferrer F, Mannis MJ. Surgical monovision and monovision reversal in LASIK. Cornea. 2006;25(2):136-138.

23. Durrie DS. The effect of different monovision contact lens powers on the visual function of emmetropic presbyopic patients (an American Ophthalmological Society thesis). Trans Am Ophthalmol Soc. 2006; 104:366-401.

24. Goldberg DB. Comparison of myopes and hyperopes after laser in situ keratomileusis monovision. J Cataract Refract Surg. 2003;29(9): 1695-1701.

25. Kruh JN, Garrett KA, Huntington B, Robinson S, Melki SA. Risk factors for retreatment following myopic LASIK with femtosecond laser and custom ablation for the treatment of myopia. Semin Ophthalmol. 2017; 32(3):316-320.
26. Ghanem RC, de la Cruz J, Tobaigy FM, Ang LP, Azar DT. LASIK in the presbyopic age group: safety, efficacy, and predictability in 40- to 69-year-old patients. Ophthalmology. 2007;114(7):1303-1310.

27. Evans BJ. Monovision: a review. Ophthalmic Physiol Opt. 2007;27(5): 417-439.

28. Jain S, Arora I, Azar DT. Success of monovision in presbyopes: review of the literature and potential applications to refractive surgery. Surv Ophthalmol. 1996;40(6):491-499.

29. Seyeddain O, Hohensinn M, Riha W, et al. Small-aperture corneal inlay for the correction of presbyopia: 3-year follow-up. J Cataract Refract Surg. 2012;38(1):35-45.

30. Yoo A, Kim JY, Kim MJ, Tchah H. Hydrogel inlay for presbyopia: objective and subjective visual outcomes. J Refract Surg. 2015;31(7): 454-460.

31. Luger MH, McAlinden C, Buckhurst PJ, Wolffsohn JS, Verma S, Arba Mosquera S. Presbyopic LASIK using hybrid bi-aspheric micromonovision ablation profile for presbyopic corneal treatments. Am J Ophthalmol. 2015;160(3):493-505.
Clinical Ophthalmology

\section{Publish your work in this journal}

Clinical Ophthalmology is an international, peer-reviewed journal covering all subspecialties within ophthalmology. Key topics include: Optometry; Visual science; Pharmacology and drug therapy in eye diseases; Basic Sciences; Primary and Secondary eye care; Patient Safety and Quality of Care Improvements. This journal is indexed on

\section{Dovepress}

PubMed Central and CAS, and is the official journal of The Society of Clinical Ophthalmology (SCO). The manuscript management system is completely online and includes a very quick and fair peer-review system, which is all easy to use. Visit http://www.dovepress.com/ testimonials.php to read real quotes from published authors. 